\title{
SÍNDROME DEL CASCANUECES. A PROPÓSITO DE UN CASO Y REVISIÓN DE LA LITERATURA
}

\author{
D. SANTOS ARRONTES, R. SALGADO SALINAS*, V. CHIVA ROBLES, \\ J.M. GÓMEZ DE VICENTE, I. FERNÁNDEZ GONZÁLEZ, J. COSTA SUBIAS*, \\ A. PÁEZ BORDA, A. BERENGUER SÁNCHEZ
}

Servicio de Urología. *Servicio de Radiodiagnóstico. Hospital Universitario de Getafe. Getafe (Madrid).

Actas Urol Esp. 27 (9): 726-731, 2003

\section{RESUMEN}

SÍNDROME DEL CASCANUECES. A PROPÓSITO DE UN CASO Y REVISIÓN DE LA LITERATURA

$\mathrm{El}$ síndrome del cascanueces está causado por una compresión de la vena renal entre la aorta y la arteria mesentérica superior. Este fenómeno resulta en una hipertensión de la vena renal izquierda, varices en la vena gonadal izquierda y hematuria unilateral.

Comunicamos un caso típico de síndrome de cascanueces y se lleva a cabo una revisión de la literatura reciente para tratar de explicar esta patología.

PALABRAS CLAVE: Síndrome cascanueces. Hematuria. Revisión.

\section{ABSTRACT}

NUTCRACKER SYNDROME. A CASE REPORT AND REVIEW OF THE LITERATURE

Nutcracker syndrome is caused by compression of left renal vein between the aorta and the superior mesenteric artery. This phenomenon results in left renal venous hypertension, left gonadal vein varices and unilateral hematuria.

We report a typical case of nutcracker syndrome and we review the literature in an effort to explain this pathology.

KEY WORDS: Nutcracker syndrome. Hematuria. Review.

$\mathrm{E}$ síndrome del cascanueces constituye una rara causa de hematuria procedente del sistema colector izquierdo secundario a compresión de la vena renal izquierda entre la arteria mesentérica superior y la aorta.

Puede aparecer en ambos sexos, su prevalencia no se conoce ya que normalmente se trata de un cuadro asintomático.

Presentamos el caso clínico y hacemos una revisión reciente de la literatura.

\section{CASO CLÍNICO}

Un varón de 18 años fue hospitalizado por hematuria persistente de 2 meses de evolución. No tenía historia personal relevante (no cólicos renoureterales, no infecciones urinarias o del tracto respiratorio superior, no ingesta de fármacos nefrotóxicos, y sin antecedentes de cirugía o trauma abdominal), ni antecedentes familiares de enfermedad renal. La exploración física fue rigurosamente normal. 
Los exámenes iniciales que incluyeron sistemático, bioquímica y coagulación sanguínea fueron normales. El sistemático de orina reveló abundantes eritrocitos por campo de gran aumento con una excreción de proteínas entre 0,5 y $1 \mathrm{~g} / \mathrm{dl}$. La citología urinaria fue negativa para malignidad y la cistoscopia evidenció sangrado procedente del uréter izquierdo.

Los anticuerpos antinucleares, anti-DNA y anti-citoplasma neutrófilo (ANCA) fueron negativos. Los niveles séricos de IgA, IgG e IgM, la concentración de complemento, la velocidad de sedimentación, ASLO y el factor reumatoide fueron normales, así como también la ecografía abdominal, la urografía intravenosa y la arteriografía.

La tomografía axial computerizada evidenció una compresión de la vena renal izquierda por la pinza aorto-mesentérica (Fig. 1) y la venografía una dilatación de la vena renal izquierda, con un gradiente de presión entre la vena renal izquierda y la vena cava inferior de $4 \mathrm{mmHg}$, siendo diagnosticado de síndrome del cascanueces (Fig. 2).

Posteriormente se realizó una ureteroscopia rígida y flexible con bomba de perfusión, aplicándose una hiperpresión de la vía urinaria. Tras doce meses de seguimiento el paciente no ha vuelto a presentar episodios de hematuria macro ni microscópica.

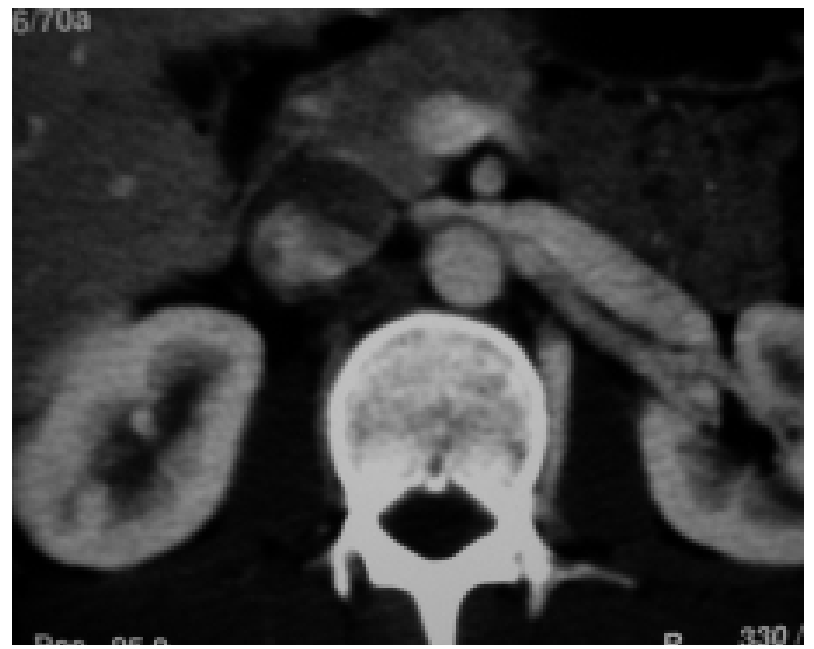

FIGURA 1. CT abdomen con c.i.v. afilamiento de vena renal izquierda en su trayecto a través de la pinza aortomesentérica. Vena lumbar izquierda prominente.

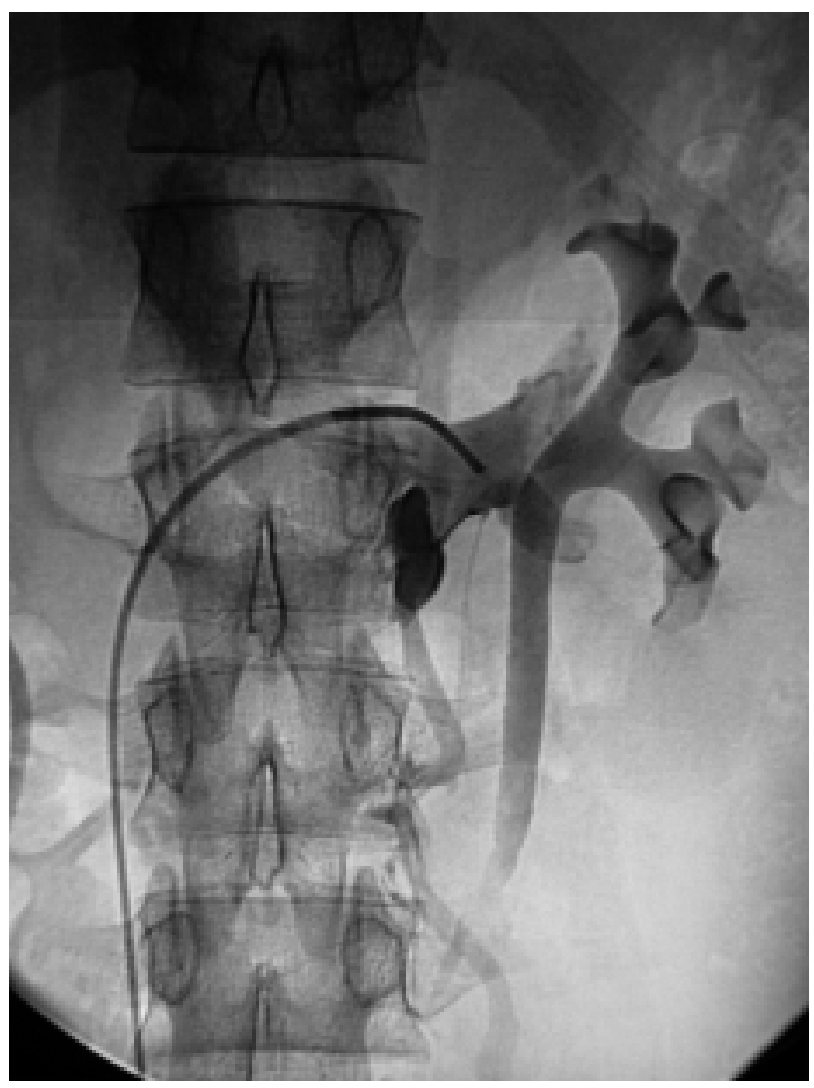

FIGURA 2. Flebografia de vena renal izquierda. Se observan venas de drenaje prominentes hacia la región lumbar. Se realizan medidas de presión en vena renal izquierda y vena cava obteniéndose un gradiente de 4 mm de $\mathrm{Hg}$.

\section{DISCUSIÓN}

El síndrome o fenómeno del cascanueces fue descrito inicialmente por De Schepper en 1972, que lo denominó como "síndrome de atrapamiento de la vena renal izquierda"1. Constituye una causa rara de hematuria procedente del sistema colector izquierdo secundaria a compresión de la vena renal izquierda entre la arteria mesentérica superior y la aorta. Esta compresión se traduce en una hiperpresión del sistema venoso renal izquierdo, con el posterior desarrollo de varicosidades a nivel de la pelvis renal y uréter, que pueden comunicarse con la vía excretora y dar lugar a episodios de hematuria ${ }^{2-4}$.

Es un cuadro clínico análogo al "síndrome de la arteria mesentérica superior", en el que la tercera porción duodenal es comprimida entre la arteria mesentérica superior y la aorta, dando lugar a un cuadro obstructivo intestinal. 
Aparece por igual en ambos sexos, y dado que muchas veces se trata de un cuadro asintomático, su verdadera prevalencia no se conoce $^{5}$. Aparece en aquellos individuos en los que en ángulo entre la arteria mesentérica superior y la aorta está disminuido. De esta forma, aquellos pacientes con ptosis renal, con escasa grasa perirrenal y lordosis lumbar acentuada presentan una predisposición anatómica. Del mismo modo, puede presentarse durante el embarazo, y además puede empeorar durante el tercer trimestre $\mathrm{e}^{6,7}$.

La presencia de fístulas arteriovenosas intrarrenales que se acompañan de un aumento de flujo a nivel de la vena renal, con hiperpresión de la misma, pueden originar lo que se denomina "pseudonutcracker effect"8, que también puede aparecer asociado a una vena renal izquierda retro-aórtica ${ }^{9}$ o a una fístula aorto-vena renal izquierda ${ }^{10}$.

Clínicamente puede permanecer silente o manifestarse como episodios de hematuria macro o microscópica ${ }^{11-13}$, que se puede acompañar de dolor en fosa renal izquierda y/o dolor abdominal. Característicamente, la hematuria (y el dolor si está presente) es más intensa con el ortostatismo, al aumentar la presión de la columna de sangre a nivel de la vena renal izquierda (síndrome doloroso de la vena gonadal) y con el ejercicio ${ }^{14}$.

También se ha relacionado con episodios de proteinuria recurrente en jóvenes ${ }^{15}$ que se incrementa con el ortostatismo ${ }^{16}$, así como con el síndrome de fatiga crónica, que debe sospecharse en aquellos niños con desórdenes psicosomáticos, hipotensión ortostática, taquicardia postural $u$ otros sintomas de disfunción autonómica ${ }^{17}$.

Puede cursar con varicocele izquierdo y varices lumbares ${ }^{18}$, debido a que el incremento de presión a nivel de la vena renal izquierda se transmite de modo retrógrado por la vena gonadal del mismo lado. Esto produce un incremento de presión a nivel del plexo pampiniforme, tributario de la vena gonadal, con el desarrollo de varicocele izquierdo en diferentes grados. Esta obstrucción al flujo gonadal también se relaciona con síntomas congestivos pelvianos. Scultetus et al. estudiaron 9 mujeres con síntomas congestivos pelvianos (dolor pélvico crónico, dispareunia, disuria y dismenorrea) y hematuria diagnosticadas de sindrome del cascanueces con gradientes renocavos mayores de $4 \mathrm{mmHg}$ (normal de 0 a 1 mmHg). La colocación de stents externos en 2 pacientes, internos en 1 y la realización de un bypass gonadocavo en tres mejoró la hematuria en el 100\% de los pacientes y se consiguió un alivio del dolor (de acuerdo con una escala numérica) del $90 \%{ }^{19}$.

Existen casos de pacientes con fenómeno de cascanueces complicados con nefropatía IgA, en cuyo caso presentan empeoramiento de la hematuria tras infecciones del tracto respiratorio superior $^{20}$, sin que pueda establecerse ninguna relación entre ambos procesos.

Se trata de una patología difícil de diagnosticar mediante métodos rutinarios. Inicialmente debe realizarse un estudio de hematuria para descartar otras causas más frecuentes. La cistoscopia nos informará de la emisión de orina hematúrica por el meato ureteral izquierdo.

La flebografía constituye la técnica de elección puesto que nos permite llevar a cabo cálculos de gradientes de presión entre la porción distal de la vena renal y la vena cava inferior. No obstante el cálculo de este gradiente no es imprescindible para el diagnóstico pudiendo presentarse variaciones del mismo en función del grado de circulación colateral existente. De esta forma, en fases muy precoces, nos podemos encontrar con una vena renal dilatada con hiperpresión, y en fases más avanzadas, una vez que se desarrolla la circulación colateral nos podemos encontrar una vena dilatada con un gradiente normal. La distinción entre una vena dilatada normal o una dilatación asociada a un síndrome de cascanueces en pacientes con una hipertensión mínima puede ser muy compleja. Por otro lado, la presencia de una dilatación de la vena renal asociada a una hipertensión de la misma en el seno de un síndrome de cascanueces nos informa que se trata de un proceso no compensado, por el contrario si existe una normopresión el proceso estará compensado $^{21}$.

Según Beinart et al. presiones en la vena renal superiores a $1 \mathrm{mmHg}$ indican hipertensión a nivel de la vena renal ${ }^{22}$. Nishimura et al. por el contrario, estudiaron nueve sujetos sanos apre- 
ciándose gradientes de hasta $3 \mathrm{mmHg}^{23}$. Por dicho motivo, Takebayashi et al. clasifican estos gradientes de presión como normales $k 1$ $\mathrm{mmHg}$ ), borderline (1-3 mmHg) e hipertensión de vena renal izquierda $(>3 \mathrm{mmHg})^{21}$.

No obstante, la flebografía es una prueba invasiva y por ello se estudian métodos menos agresivos como son la ecografía, la ecografía-doppler, el TAC y la MR.

Okada et al. estudiaron mediante ecografía 125 niños divididos en tres grupos que incluían 23 con hematuria macroscópica, 52 con hematuria microscópica y 50 controles. Los criterios de exclusión fueron la presencia de nefritis, litiasis o tumor. Calcularon el ratio entre el diámetro de la parte dilatada de la vena renal izquierda con la parte estrecha de la misma y el diámetro aórtico respectivamente, así como la diferencia de diámetros entre la vena renal izquierda y derecha. Los puntos de corte establecidos fueron de 3.7, 0.75 y $1.7 \mathrm{~mm}$, estableciéndose el diagnóstico de sindrome de cascanueces cuando al menos dos de estos parámetros se encuentran por encima del punto de corte ${ }^{24}$.

La ecografía doppler nos permite determinar el diámetro y velocidad máxima de flujo en las porciones proximal y distal de la vena renal izquierda, aunque en la primera de ellas su determinación es complicada debido al artefacto de la arteria mesentérica superior y de la aorta. Un ratio distal/proximal $>5$ es diagnóstica de síndrome de cascanueces, aunque pueden perderse aquellos pacientes con un síndrome de larga evolución en los que la vena renal no está distendida ${ }^{25}$.

Takebayashi et al. compararon la ecografía doppler con la venografía en 44 pacientes con síndrome del cascanueces, estableciendo una sensibilidad y especificidad de la ecografía doppler para el diagnóstico del mismo en el $78 \mathrm{y}$ $100 \%$ respectivamente ${ }^{21}$.

Dada la utilidad de la ecografía doppler y su inocuidad, algunos autores consideran que puede tratarse de una herramienta útil en el screening de la proteinuria ortostática para evaluar si la causa es un síndrome del cascanue$\operatorname{ces}^{26}$.

El CT aporta información anatómica sobre la relación de la vena renal izquierda con la aorta y la arteria mesentérica superior pudiendo estable- cer el diagnóstico de síndrome de cascanue$\operatorname{ces}^{27,28}$. Algunos autores han sugerido que si el cociente entre el diámetro de la vena renal izquierda proximal y distal al ángulo aortomesentérico es igual o superior a 1,5 se trata de un fenómeno de cascanueces. No obstante Zerín et al., estudiaron 39 pacientes con edad media de 10,6 años sin hematuria, encontrando que 22 $(51,3 \%)$ presentaban un cociente superior a 1,5 , por lo que este valor no puede ser utilizado como referencia pues se trata de una variante de la normalidad $^{29}$.

Menor experiencia existe actualmente con la RM para el diagnóstico del síndrome de cascanueces, aunque podría tratarse de una prueba válida para el diagnóstico ${ }^{30-32}$.

El tratamiento del síndrome del cascanueces depende de la severidad del sangrado. Aquellos pacientes que presentan hemorragias intermitentes sin anemización no requerirán ningún tipo de tratamiento, excepto suplementos orales de hierro si fuese preciso. Aquellos casos que se acompañen de dolor en flanco izquierdo incapacitante, hematuria macroscópica frecuente o anemización pueden requerir un tratamiento más agresivo que incluye técnicas quirúrgicas; dentro de las cuales cabe citar el autotransplante ${ }^{27,33}$ y la trasposición de la vena renal izquierda ${ }^{34,35}$. Ahora bien, en el segundo caso, en aquellos pacientes en los cuales ya se haya establecido un shunt entre la vena renal y el sistema colector la hematuria puede persistir tras la intervención.

Se ha investigado la realización de angioplastias con balón ${ }^{36}$ o la implantación de stents ${ }^{37,38}$, con resultados favorables y menor morbilidad, aunque su eficacia y seguridad debe ser comprobada a largo plazo.

La ureteropieloscopia en el diagnóstico diferencial de hematuria del tracto urinario superior de etiología desconocida juega un papel importante ${ }^{39,40}$; no obstante su utilidad en el síndrome del cascanueces no está establecido. En el caso que se presenta, tras la realización de una ureteroscopia flexible con bomba de perfusión, creando una hiperpresión de la vía urinaria, cesó el cuadro hematúrico. No obstante se requieren más estudios para determinar la existencia de una posible relación entre ambos fenómenos. 


\section{REFERENCIAS}

1. DE SCHEPPER A.: Nutcracker phenomenon of the left renal vein pathology. J Belg Rad 1972; 55: 507511 .

2. LIDOVE O, OROZCO R, GÚCRY B, CORREAS JM, ROBINO C, MÉJEAN A.: A young woman with intermittent macroscopic haematuria. Nephrol Dial Transplant 2001; 16: 853-855.

3. TRAMBERT JJ, RABIN AM, WEISS KL, TEIN AB.: Pericaliceal varices due to the nutcracker phenomenon. AJR Am J Roentgenol 1990 feb; 154 (2): 305-306.

4. MURAYAMA S, SHIMODA Y, KISHIKAWA T.: Pyelovenous backflow in left renal vein hypertension: case report. Radiat Med 1989 mar-apr; 7 (2): 55-57.

5. CHEN YM, WANG IK, NG KK, HUANG CC.: Nutcracker syndrome: an overlooked cause of hematuria. Chang Gung Med J 2002 oct; 25 (10): 700-705.

6. ITOH S, YOSHIDA K, NAKAMURA Y, MITSUHASHI N.: Aggravation of the nutcracker syndrome during pregnancy. Obstet Gynecol 1997 oct; 90 (4 Pt 2): 661-663.

7. UZU T, KO M, YAMATO M, TAKAHARA K, YAMAUCHI A.: A case of nutcracker syndrome presenting with hematuria in pregnancy. Nephron 2002 aug; 91 (4): 764-765.

8. YAGMURLU B, OZCAN H, AYTAC SK, BILGIC S, SANLIDILEK U.: Intrarenal arteriovenous fistula causing a "pseudonutcracker effect". J Clin Ultrasound $2002 \mathrm{feb} ; 30$ (2): 109-113.

9. GIBO M, ONITSUKA H.: Retroaortic left renal vein with renal vein hypertension causing haematuria. Clin Imaging 1998 nov-dec; 22 (6): 422-424.

10. MANSOUR MA, RUTHERFORD RB, METCALF RK, PEARCE WH.: Spontaneous aorto-left renal vein fistula: the "abdominal pain, hematuria, silent left kidney" syndrome. Surgery 1991 jan; 109 (1): 101 106.

11. RUSSO D, MINUTOLO R, IACCARINO V, ANDREUCCI M, CAPUANO A, SAVINO FA.: Gross hematuria of uncommon origin: the nutcracker syndrome. Am J Kidney Dis 1998 sep; 32 (3): E3.

12. SHAPER KR, JACKSON JE, WILLIAMS G.: The nutcracker syndrome: an uncommon cause of haematuria. Br J Urol 1994 aug; 74 (2): 144-146.

13. LEE CC, LIN JT, DENG HH, LIN ST.: Hematuria due to nutcracker phenomenon of left renal vein: report of a case. J Formos Med Assoc 1993 mar; 92 (3): 291-293.

14. HANNA HE, SANTELLA RN, ZAWADA ET Jr, MASTERSON TE.: Nutcracker syndrome: an underdiagnosed cause for hematuria? S D J Med $1997 \mathrm{dec}$; 50 (12): 429-436.

15. FAIZAN MK, FINN LS, PALADIN AM, MCDONALD RA.: A 14-year-old girl with recumbent proteinuria. Pediatr Nephrol 2002 may; 17 (5): 379-381.

16. LEE SJ, YOU ES, LEE JE, CHUNG EC.: Left renal vein entrapment syndrome in two girls with orthostatic proteinuria. Pediatr Nephrol 1997 apr; 11 (2): 218-220.
17. TAKAHASHI Y, OHTA S, SANO A, KURODA Y, KAJI Y, MATSUKI M, MATSUO M.: Does severe nutcracker phenomenon cause pediatric chronic fatigue? Clin Nephrol 2000 mar; 53 (3): 174-181.

18. LITTLE AF, LAVOIPIERRE AM.: Unusual clinical manifestations of the nutcracker syndrome. Australas Radiol 2002 jun; 46 (2): 197-200.

19. SCULTETUS AH, VILLAVICENCIO JL, GILLESPIE DL.: The nutcracker syndrome: its role in the pelvic venous disorders. J Vasc Surg 2001 nov; 34 (5): 812-819.

20. OZONO Y, HARADA T, NAMIE S, ICHINOSE H, SHIMAMINE R, NISHIMAWA Y, HARA K.: The "nutcracker" phenomenon in combination with IgA nephropathy. J Int Med Res 1995 mar-apr; 23 (2): 126131.

21. TAKEBAYASHI S, UEKI T, IKEDA N, FUJIKAWA A.: Diagnosis of the nutcracker syndrome with color doppler sonography: correlation with flow patterns on retrograde left venal venography. Am J Roentgenol 1999; 172 (1): 39-43.

22. BEINART C, SNIDERMAN KW, TAMURA S, VAUGHAN ED, SOS TA.: Left renal vein to inferior vena cava pressure relationship in humans. J Urol 1981; 127: 1070-1071.

23. NISHIMURA Y, FUSHIKI M, YOSHIDA M et al.: Left renal vein hipertensión in patients with left renal bleeding of unknown origin. Radiology 1986; 160: 663-667.

24. OKADA M, TSUZUKI K, ITO S.: Diagnosis of the nutcracker phenomenon using two-dimensional ultrasonography. Clin Nephrol 1998 jan; 49 (1): 3540.

25. KIM SH, CHO SW, KIM HD, CHUNG JW, PARK JH, HAN MC.: Nutcracker syndrome: diagnosis with doppler US. Radiology 1996; 198: 93-97.

26. PARK SJ, LIM JW, CHO BS, YOON TY, OH JH.: Nutcracker syndrome in children with orthostatic proteinuria: diagnosis on the basis of Doppler sonography. J Ultrasound Med 2002 jan; 21 (1): 3945; quiz 46.

27. SHOKEIR AA, EL-DIASTY TA, GHONEIM MA.: The nutcracker syndrome: new methods of diagnosis and treatment. Br J Urol 1994 aug; 74 (2): 139143.

28. KANEKO K, KIYA K, NISHIMURA K, SHIMIZU T, YAMASHIRO Y.: Nutcracker phenomenon demonstrated by three-dimensional computed tomography. Pediatr Nephrol 2001; 16: 745-747.

29. ZERIN JM, HERNÁNDEZ RJ, SEDMAN AB, KELSCH RC.: "Dilatation" of the left renal vein on computed tomography in children: a normal variant. Pediatr Radiol 1991; 21 (4): 267-269.

30. KANEKO K, OHTOMO Y, YAMASHIRO Y, OBINATA K, KUROKAWA S, AIZAWA S.: Magnetic resonance angiography in nutcracker phenomenon. Clin Nephrol 1999 apr; 51 (4): 259-260.

31. TAKEMURA T, IWASA H, YAMAMOTO S, HINO S, FUKUSHIMA K, ISOKAWA S, OKADA M, YOSHIOKA K.: Clinical and radiological features in four adolescents with nutcracker syndrome. Pediatr Nephrol 2000 sep; 14 (10-11): 1002-1005. 
32. HOHENFELLNER M, STEINBACH F, SCHULTZLAMPEL D, SCHANTZEN W, WALTER K, CRAMER BM, THUROFF JW, HOHENFELLNER R.: The nutcracker syndrome: new aspects of pathophysiology, diagnosis and treatment. J Urol 1991 sep; 146 (3): 685-688.

33. CHUANG CK, CHU SH, LAI PC.: The nutcracker syndrome managed by autotransplantation. J Urol 1997; 157: 1833-1834.

34. HOHENFELLNER M, D'ELIA G, HAMPEL C, DAHMS S, THUROFF JW.: Transposition of the left renal vein for treatment of the nutcracker phenomenon: long-term follow-up. Urology 2002 mar; 59 (3): 354-357.

35. STEWART BH, REIMAN G.: Left renal venous hypertension "nutcracker" syndrome. Managed by direct renocaval reimplantation. Urology 1982 oct; 20 (4): 365-369.

36. TAKAHASHI Y, SANO A, MATSUO M.: An effective "transluminal balloon angioplasty" therapy for pediatric chronic fatigue syndrome with nutcracker phenomenon. Clin Nephrol 2000 jan; 53 (1): 77-88.

37. SEGAWA N, AZUMA H, IWAGOTO $\mathrm{Y}$ et al.: Expandable metallic stent placement for nutcracker phenomenon. Urology 1999; 53: 631-633.
38. CHIESA R, ANZUINI A, MARONE EM, BRIGUORI C, MOURA MR, MELISSANO G, COLOMBO A, ROSANIO S.: Endovascular stenting for the nutcracker phenomenon. $J$ Endovasc Ther 2001 dec; 8 (6): 652-655.

39. KUMON H, TSUGAWA M, MATSUMURA Y, OHMORI H.: Endoscopic diagnosis and treatment of chronic unilateral haematuria of uncertain etiology. $J$ Urol 1990 mar; 143 (3): 554-558.

40. YAZAKI T, KAMIYAMA Y, TOMOMASA H, SHIMIZU $\mathrm{H}$, OKANO Y, IIYAMA T, IIZUMI T, UMEDA T.: Ureteropyeloscopy in the diagnosis of patients with upper tract haematuria: an initial clinical study. Int J Urol 1999 may; 6 (5): 219-225.

\section{Dr. D. Santos Arrontes}

Servicio de Urología

Hospital Univ. de Getafe

Ctra. de Toledo km. 12,500

28905 Getafe (Madrid)

(Trabajo recibido el 21 abril de 2003) 